\title{
Une nouvelle espèce de Trichoptère du Sud de l'Espagne : Oecetis grazalemae (Trichoptera : Leptoceridae)
}

\author{
M. A. González: 1 \\ J. C. Iglesiast
}

Mots clés : Trichoptera, Leptoceridae, nouvelle espece, Espagne.

Description de Oecelis grazalemae, nouvelle espèce du Sud de l'Espagne, qui est comparée avec les espèces voisines.

A new species from South Spain : Oecetis grazalemae (Trichoptera : Ieptoceridae).

Keywords : Trichoptera, Leptoceridae, new species, Spain.

Oecetis grazalemae, a new species from south Spain is described and compared with closely related species.

La faune des Trichoptères du sud de la Péninsule ibérique est encore très mal connue (González et al. (1987). Des recherches poursuivies ces dernières années dans cette région par différents auteurs ont abouti à la découverte de nombreuses espèces nouvelles; dans le cadre de nos recherches sur la faune de l'Andalousie occidentale nous présentons ici la description d'un nouveau Leptoceridae du genre Oeceris McLachlan.

\section{Oecetis grazalemae n. sp.}

Matériel : holotype mâle, Espagne, Puente de la Terrona (Cádiz), rio Guadalalete, $360 \mathrm{~m}$, 7-VIII-86 (leg. Valiela J. et Sosa E.), coll. González (Departamento de Biología Animal, Universidad de Santiago).

\section{DESCRIPTION DU MALE}

Coloration générale du corps jaunâtre (exemplaire conservé en alcool) ; thorax brun pâle ; ailes à membrane pâle et à peu près de la même largeur. Longueur de l'aile antérieure : $8,2 \mathrm{~mm}$; de l'aile postérieure: $6,3 \mathrm{~mm}$. Nervation typique pour le gen re (voir Fig. 6). Eperons 1, 2, 2.

\footnotetext{
1. Departamento de Biología Animal (Zoología), Facultad de Bio logia, Universidad de Santiago de Compostela, Santiago, España.
}

VIII' tergite sans réticulé visible. IX'e segment sensiblement plus long, ventralement que dorsalement ; le bord antérieur est nettement bombé à sa moitié ventrale : à sa partie la plus dorsale le IX e segment est réduit à une étroite bande à peu près verticale : sur la partie medio-distale du IX $\mathrm{X}^{\mathrm{e}}$ tergite il y a une

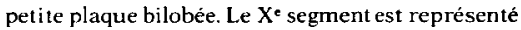
par un lobe médian impair en bande étroite, légèrement élargie à sa partie basale et dirigée obliquement vers le bas, et une paire de lobes triangulaires, très hyalins, leurs apex servant de points d'insertion à une petite soie. Appendices préanaux, en vue latérale, en forme de bandes rectangulaires obliquement découpées à l'apex et environ trois fois plus longues que larges. Appendices inférieurs assez grands, subrectangulaires vus de profil, avec le bord supérieur légèrement convexe et l'angle distalinférieur prolongé en une sorte de lobe arrondi ; vus de dessous ils se mont rent nettement convergents, leur apex visiblement recourbé en bec vers la ligne médiane. Appareil phallique plutôt court, recourbé vers le bas; il est composé d'une phallotèque dont la partie distale est nettement retrécie en bande grêle (en vue latérale), puis épaissie en pommeau à l'apex, et d'une endothèque spinifère qui porte, de la base vers l'apex, deux longues et grosses épines, de taille semblable, fort recourbées et mème tordues 

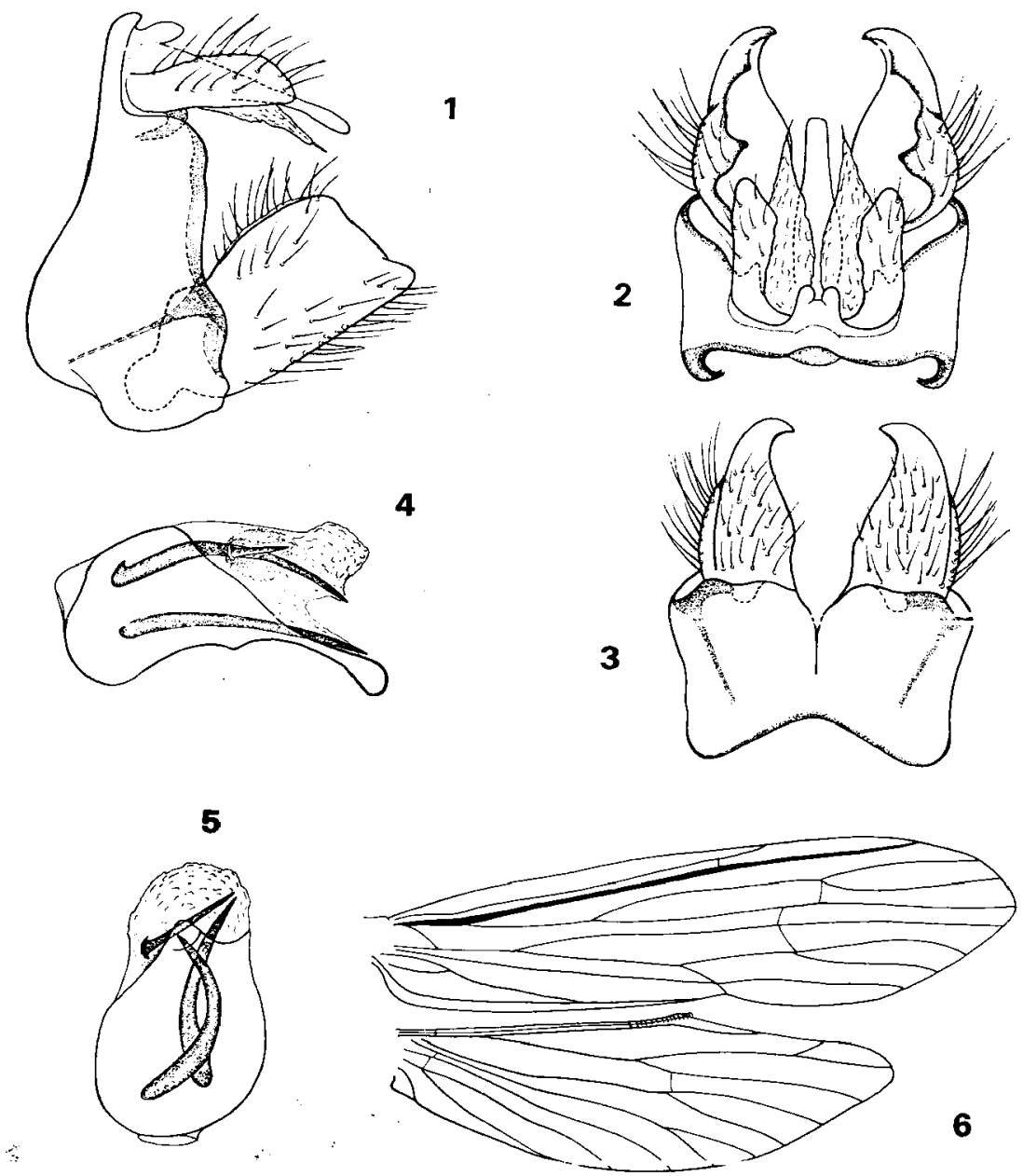

Fig. 1.6: Oecetis grazalemae n. sp. (holotype). 1 : genitalia mâle, vue latérale $: 2$ : genitalia mâle, vue dorsale ; 3 : appendices inférieurs et $\mathrm{LX}^{e}$ segment, vue ventrale ; 4 : phallus, vue latérale; $5:$ phallus, vue ventrale; $6:$ ailes. 
(ce qui est bien apparent en vue ventrale ou dorsale) et une autre distale plus courte et droite.

\section{AFFINITES}

Oecetis grazalemae sp. n. ressemble à $O$. notata (Rambur, 1842), mais elle est surtout voisine de $O$. villosa Kimmins, 1963 - espèce décrite d'Assam (Inde), voir Kimmins (1963) : figs. 115-120 -, de par l'aspect général de toutes les parties de l'armature génitale mâle ; cependant les deux espèces se diffé. rencient nettement par la forme des appendices inférieurs en vue ventrale, par la forme (vues latérale et ventrale) de la partie distale de la phallothèque et par l'armature d'épines du phallus, plus simple chez $O$. villosa ("... membrane containing a large and a small spine "d'après Kimmins). D'autres différences se situent au niveau du lobe médian du $\mathrm{X}^{\mathrm{e}}$ segment (plus long et grêle chez $O$. villosa), des lobes triangulaires hyalins de ce même segment (apparemment absents dans cette espece), de l'aspect des appendices préanaux en vue latérale et $\mathrm{du} \mathrm{IX}^{\mathrm{e}} \mathrm{seg}$ ment et l'absence de réticulé sur les tergites VII et VIII chez notre espèce.

\section{Remerciements}

Nous tenons à remercier ici le $\mathrm{D}^{r} \mathrm{H}$. Malicky qui nous a aidé pour la réalisation de ce travail.

\section{Travaux cités}

González (M.), Garcia de Jalón (D.) \& Terra (L.). 1987. — Faunistic studies on Iberian Trichoptera : A historical survey and present state of knowledge. Proc. $5 \mathrm{~h} / \mathrm{ht}$. Symp. Trichoptera. Dr. Junk Pub. Dordrecht : 85-90.

Kimmins (D.E.). 1963. - On the Leptoceridae of the Indian subcontinent and north east Burma (Trichoptera). Bull. Brit. Mus. (Nat. Hist.) Ent., 14 (6) : 263-316. 